\title{
A Review of Calphad Modeling of Ordered Phases
}

\author{
Bo Sundman ${ }^{1} \cdot$ Qing Chen ${ }^{2} \cdot$ Yong $\mathrm{Du}^{3}$
}

Submitted: 7 June 2018/in revised form: 17 July 2018/Published online: 20 August 2018

(c) ASM International 2018

\begin{abstract}
The models used in Calphad to describe long and short range ordering in multicomponent alloys have improved significantly over the last 20 years. For long range ordering the compound energy formalism has gained universal acceptance and it is now possible to calculate realistic phase diagrams including also short range ordering which means the Gibbs energy is modeled correctly. There is still a problem in separating enthalpy and entropy which makes extrapolations to low temperatures uncertain. In this paper some of the history will be reviewed together with the current status and some new ideas.
\end{abstract}

Keywords Calphad · modeling · order/disorder · phase diagrams

\section{Introduction}

In the Calphad method each phase in a system is modeled with a separate Gibbs energy expression which is a function of its constitution, $T$ and $P$. These models contain

A tribute to professor Zhanpeng Jin on his 80 birthday.

This invited article is part of a special issue of the Journal of Phase Equilibria and Diffusion in honor of Prof. Zhanpeng Jin's 80th birthday. The special issue was organized by Prof. Ji-Cheng (JC) Zhao, The Ohio State University; Dr. Qing Chen, Thermo-Calc Software AB; and Prof. Yong Du, Central South University.

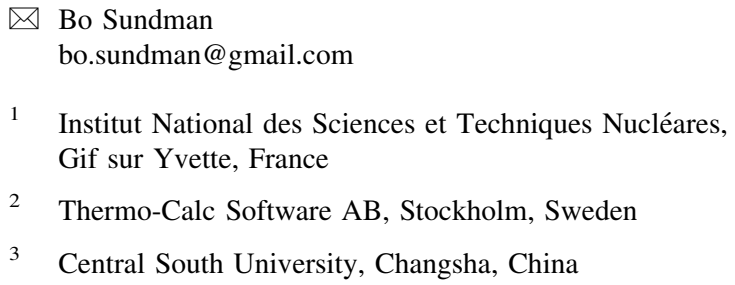

parameters that are fitted to many different kinds of experimental or theoretical data. For crystalline phases the structure is a very important part of the model. When atoms are on different lattice sites, the long range ordering (LRO), can be described by sublattices. But when the same atom can occupy different types of sites there can also be locally short range ordering (SRO) and this is more challenging to describe. For liquids or amorphous phases there are no fixed positions for the atoms but they can have short range ordering which is frequently described with similar models as used for crystalline phases.

Describing LRO and SRO has been a challenge ever since Shockley calculated his phase diagram for FCC ordering in $1938^{[1]}$ using a Bragg-Williams configurational entropy. ${ }^{[2]}$ Not until Kikuchi developed the Cluster Variation Method (CVM) in $1951^{[3]}$ the problem of modeling SRO was solved at least theoretically. The mathematical complexities of the CVM has not yet made it possible to use in any commercial database. Many of the models discussed here are explained in more detail in the book by Lukas et al. ${ }^{[4]}$

\subsection{The Generic Gibbs Energy Expression}

All Calphad type models have an explicit Gibbs energy expression for each phase $\alpha$ which consists of several parts

$G_{M}^{\alpha}={ }^{\text {srf }} G_{M}^{\alpha}-T^{\text {cfg }} S_{M}^{\alpha}+{ }^{E} G_{M}^{\alpha}+{ }^{\text {phy }} G_{M}^{\alpha}$

where ${ }^{\operatorname{srf}} G_{M}^{\alpha}$ is the surface of reference relative to other phases but also for internal ordering, $T$ is the absolute temperature, ${ }^{\mathrm{cfg}} S_{M}^{\alpha}$ is the configurational entropy, ${ }^{E} G_{M}^{\alpha}$ is the excess Gibbs energy and phy $G_{M}^{\alpha}$ is the contribution to the Gibbs energy due to specific physical phenomena like ferromagnetism. This has been explained in Lukas et al. ${ }^{[4]}$ 
and will not be discussed here. The phase superscript will only be used when necessary.

Note that all terms have a subscript $M$ to indicate that the property is per mole formula unit of the phase. A lower case $m$ is used for a property per mole of components. This distinction is important when the model include vacancies i.e. empty lattice sites.

\subsection{The Substitutional Regular Solution}

To have a baseline we start by the Gibbs energy expression for a substitutional regular solution model for a multicomponent system:

$$
G_{m}=\sum_{i} x_{i}{ }^{\circ} G_{i}+R T \sum_{i} x_{i} \ln \left(x_{i}\right)+{ }^{E} G_{m}+{ }^{\text {phy }} G_{m}
$$

where $x_{i}$ is the mole fraction of component $i,{ }^{\circ} G_{i}$ is the Gibbs energy of component $i$ relative to the same standard state, $R$ is the gas constant and $x_{i} \ln \left(x_{i}\right)$ is the ideal entropy of mixing. Note that in this case we use the property per mole of components. The pre-superscript in ${ }^{\circ} G_{i}$ means it is for a pure component and may depend on $T$ and $P$ but not on composition. We will later use the same symbol, but without the "o", for the partial Gibbs energy calculated from a model.

\subsubsection{The Excess Gibbs Energy}

The excess Gibbs energy will in general contain several terms that depend on the composition and $T$. The composition dependence is basically a series expansion of the phase constitution:

${ }^{E} G_{m}=\sum_{i} \sum_{j>i} x_{i} x_{j}\left(L_{i j}+\sum_{k>j} x_{k}\left(L_{i j k}+\cdots\right)\right)$

There are very few cases with parameters involving more than 3 components. The binary $L_{i j}$ can depend on composition and $T$. There are several formulations of the composition dependence and the most used one is the RedlichKister polynomial ${ }^{[5]}$ because it is symmetrical:

$L_{i j}=\sum_{v=0}\left(x_{i}-x_{j}\right)^{v} \cdot{ }^{v} L_{i j}$

where the parameters ${ }^{v} L_{i j}$ may depend linearly on $T$. Only if there are significant amount of experimental heat capacity data the excess parameters may include a $T \ln (T)$ term. In a few cases the ternary parameter $L_{i j k}$ is composition dependent as described in Ref 4.

\subsubsection{The Partial Gibbs Energy and the Chemical Potential}

The chemical potential for a component $i$, denoted $\mu_{i}$ is an important property of a system and at equilibrium this is the same as the partial Gibbs energy which can be calculated from the substitutional regular solution model as:

$$
\begin{aligned}
G_{i}= & \left(\frac{\partial G}{\partial N_{i}}\right)_{T, P, N_{j \neq i}}=G_{m}+\left(\frac{\partial G_{m}}{\partial x_{i}}\right)_{T, P, x_{j} \neq i} \\
& -\sum_{j} x_{j}\left(\frac{\partial G_{m}}{\partial x_{j}}\right)_{T, P, x_{k} \neq j}
\end{aligned}
$$

A derivation of this formula is found in Ref 4 and 6 . At equilibrium, stable or metastable, the value calculated by Eq 5 must be the same as the chemical potential, $\mu_{i}$, for element $i$ in all stable phases.

\section{Long Range Ordering}

In a substitutional phase all components occupy the same set of sites. In order to have LRO a phase must have several distinct sublattices for the components, and some of the sublattices may be preferred by certain components. The lattice gives a regular 3D arrangement of atoms.

There are two very different types of LRO. In the most common case the phase will always have LRO because the sublattices are crystallographically different, for example the $\sigma$ phase with 5 different sublattices or the Laves phase with two or more. The other type of LRO can occur in phases with very simple lattices like FCC, BCC or HCP where at certain ranges of $T$ and composition the atoms arrange themselves to have a specific kind of atom as the nearest neighbour, like B2 ordering in Al-Fe. This ordering can disappear when $T$ or the composition is changed and this is known as an order/disorder transformation. In some cases it is of first order but it can also be of second order without any two-phase region. It is thus necessary to model both the ordered and disordered state with the same Gibbs energy function. Some of the crystalline structures discussed in this paper are shown in Fig. 1.

Phases which are always ordered can have different degrees of ordering and approach an almost disordered state but they can never be completely disordered. By contrast, for a model of a B2 ordered phase we must take into account that it must also be able to describe the completely disordered state where all lattice sites are equivalent. 
A1

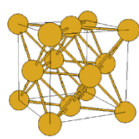

A2

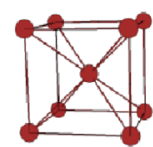

$\mathrm{D} 0_{3}$,
B1

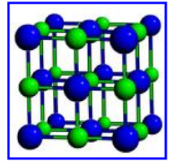

B2
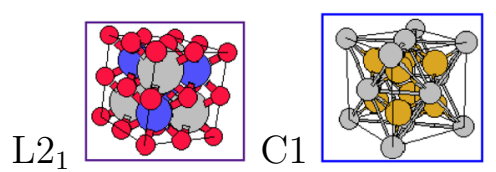
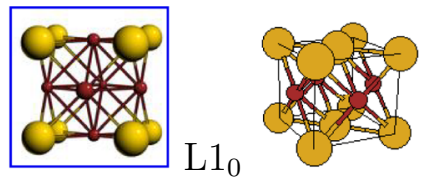

A12
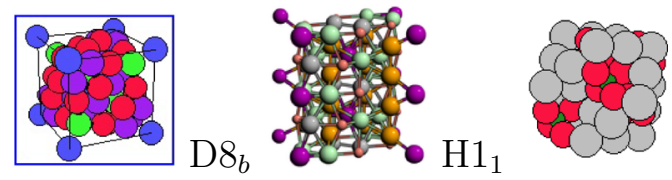

Fig. 1 Some common lattices from left to right on top row: $\mathrm{FCC}, \mathrm{BCC}, \mathrm{NaCl}, \mathrm{CsCl}, \mathrm{Ni}_{3} \mathrm{Al}, \mathrm{AuCu}$. On second row: $\mathrm{Fe}_{3} \mathrm{Al}, \mathrm{Heusler} \mathrm{Ca}_{2} \mathrm{~F}, \chi, \sigma$, spinel

\subsection{The Constitution Variable, $y_{i s}$}

In order to describe the constitution of a phase with several sublattices the mole fraction is not enough, we need another type of composition variable, the constituent or sublattice fraction $y_{j s}$ where $j$ specifies the constituent and $s$ the sublattice. When there are just two sublattices we often use $y_{i}^{\prime}$ and $y_{i}^{\prime \prime}$ but with 3 or more sublattices that becomes cumbersome.

Some components may dissolve only on a single sublattice and other components can be on several sublattices. In a sublattice of some oxides and similar phases we may even have molecules or ions as constituents.

\subsubsection{Ordering Effects on the Heat Capacity}

When the constitution of a phase changes at constant composition that gives a contribution to the heat capacity. This can have significant effect and is useful to detect order/disorder phase transformations as shown for the BCC phase in the Al-Fe system in Fig. 2.

The primary goal of the Calphad modeling is to describe the phase diagram as well as all properties that can be obtained from the Gibbs energy and its first and second derivatives using a single Gibbs energy function for each phase. It is worth noting that many other phase-based properties $^{[8]}$ have also been modeled using the Calphad approach.

\subsection{The Compound Energy Formalism}

The sublattice model has a long history but only when computers became easily available and sufficiently fast there was an interest to develop mathematical models beyond the regular solution. It is an interesting fact that when Hillert et al. ${ }^{[9]}$ first modeled interstitial solutions of $\mathrm{C}$ in Fe he based his expression on a two-sublattice model by Temkin $^{[10]}$ which had been developed for molten salts. Later this was generalized by Sundman and $\AA_{\text {gren }}^{[6]}$ and turned into a formalism, the Compound Energy Formalism
(CEF), by Hillert ${ }^{[11]}$ because its mathematical expression contained a whole range of separate models for gases, the regular solution, interstitial solutions, $\mathrm{L1}_{2}$ ordered $\mathrm{Al}-\mathrm{Ni}$, topological close-packed (TCP) phases like $\sigma$ to spinel and perovskite phases with ionic constituents.

The reason for the name, compound energy, was that when we have a phase with several sublattices with different constituents it is sometimes not possible to separate the properties of individual components in this phase. Instead we must consider compounds with a specific constituent on each sublattice and use these to create the Gibbs energy expression. Such a compound is called an endmember of a phase because the phase can exist with just a single endmember and when the composition varies the fractions of different endmember varies. In some endmembers a sublattice may be vacant and the vacancy was introduced in the modeling as a real constituent with no mass or mole fraction but with its chemical potential at equilibrium always equal to zero. The relation between the mole fraction of a component and the constituent fractions in a CEF phase is given by:

$x_{i}=\frac{\sum_{s} a_{s} \sum_{j} b_{i j} y_{j s}}{\sum_{s} a_{s} \sum_{k} \sum_{j} b_{k j} y_{j s}}$

where $b_{i j}$ is the stoichiometric factor of component $i$ in constituent $j$ and $a_{s}$ is the number of sites on sublattice $s$. The fraction of vacancies is automatically excluded from the summation as the vacancy is not a component although it may be a constituent and contribute to the configurational entropy.

Some of the terms of Eq 1 in CEF can be written as:

$$
\begin{aligned}
{ }^{\operatorname{srf}} G_{M} & =\sum_{I} \Pi_{I}\left(y_{i \in I_{s}}\right){ }^{\circ} G_{I} \\
{ }^{\mathrm{cfg}} S_{M} & =-R \sum_{s} a_{s} \sum_{i} y_{i s} \ln \left(y_{i s}\right)
\end{aligned}
$$

where $I$ represents an endmember or compound or "constituent array" with one constituent in each sublattice, $\Pi_{I}\left(y_{i \in I_{s}}\right)$ is the product of one constituent fraction from each sublattice as specified by $I$ and ${ }^{\circ} G_{I}$ is the Gibbs energy of formation of the endmember $I$. The 


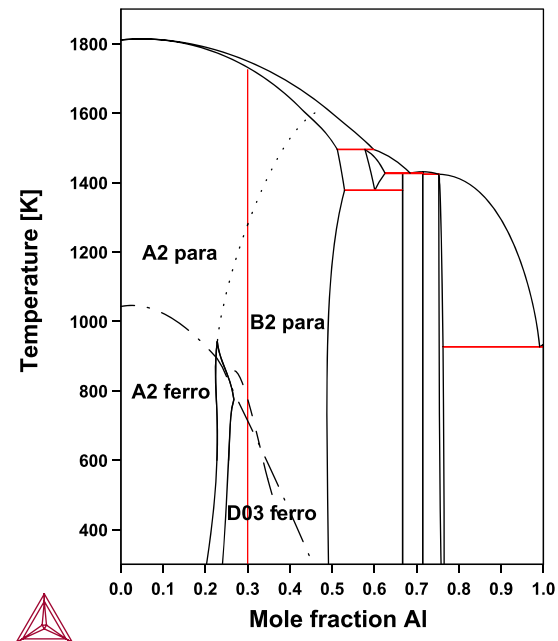

(a)

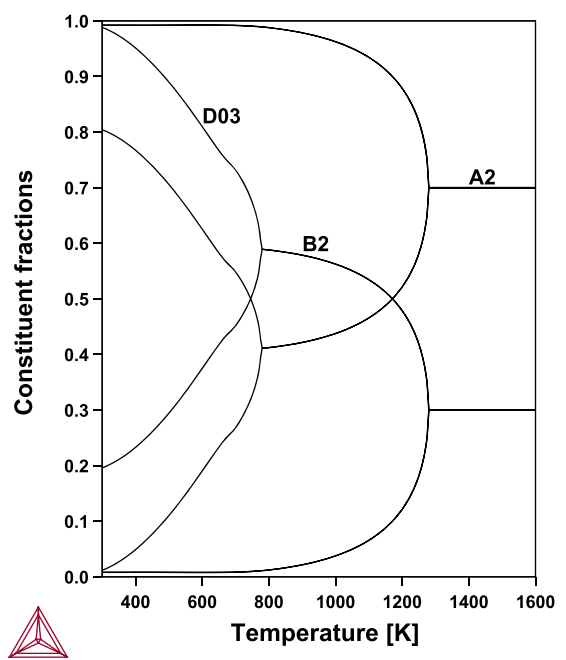

(b)

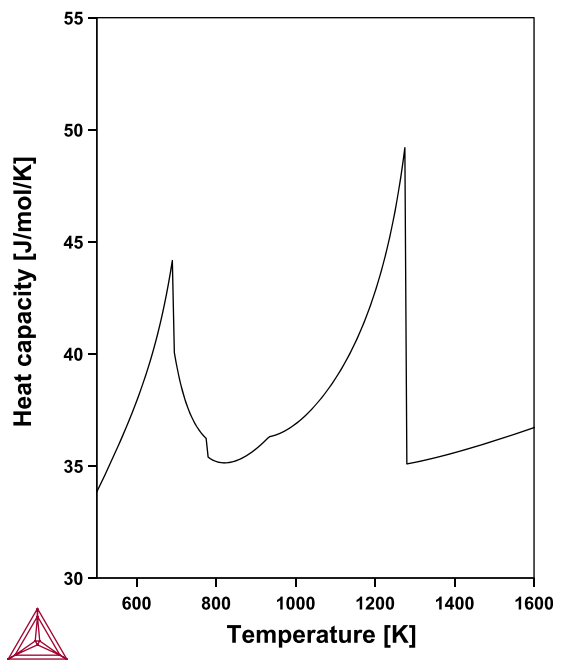

(c)
Fig. 2 The phase diagram of Al-Fe assessed by Ref 7 in (a) has several first and second order order/disorder transitions in the BCC (A2) phase. In (b) the constituent fractions in the $\mathrm{D}_{3}, \mathrm{~B} 2$ and disordered A2 phase at $30 \% \mathrm{Al}$ (along the red line in (a)) is shown as a function of $\mathrm{T}$. In (c) the effect of these ordering transformations on

configurational entropy assumes random mixing on each sublattice. The vacancy can be a constituent of any sublattice and thus contribute to the configurational entropy. For more details of the excess Gibbs energy and other terms the reader is refered to Ref 4 .

\subsubsection{The Partial Gibbs Energy Again}

When a phase is modeled with sublattices it may not be possible to calculate the partial Gibbs energy for a component. But it will always be possible to calculate the chemical potential of an endmember, $I$, using this formula

$$
\begin{aligned}
G_{I} & =G_{M}+\sum_{s}\left(\frac{\partial G_{M}}{\partial y_{i \in I_{s}}}\right)_{T, P, y_{j \neq i}} \\
& -\sum_{s} \sum_{j} y_{j s}\left(\frac{\partial G_{M}}{\partial y_{j}}\right)_{T, P, y_{k \neq j}}
\end{aligned}
$$

where $i \in I_{s}$ means the constituent in sublattice $s$ of the endmember $I$. This equation is derived in Ref 6 and 12. By combining chemical potentials of different endmembers we can usually obtain the chemical potential of the components and using the algorithm described in Ref 13 and 14 for the equilibrium calculation there is no need to explicitly calculate the chemical potential of the components for each phase, it is sufficient to calculate the first and second derivatives of the Gibbs energy with respect to T, P and the constituent fractions.

Most crystalline phases as shown in Fig. 1 have crystallographically different sites, it is only simple structures the heat capacity for the $30 \% \mathrm{Al}$ composition is shown. There is also a ferromagnetic transition which has a minor effect. All 3 figures are calculated from a single Gibbs energy function for the BCC phase with parameters which has been fitted to experimental data (Color figure online)

such as FCC, BCC and HCP that can be completely disordered.

\subsection{Phases Which are Always Ordered}

\subsubsection{Interstitial Solutions}

In the $\mathrm{C}-\mathrm{Fe}$ system carbon dissolves at the octahedral interstitial sites in FCC, which originally are empty, and in CEF the model is denoted $(\mathrm{Fe})_{1}(\mathrm{C}, \mathrm{Va})_{1}$ with $\mathrm{Fe}$ on the substitutional sites and $\mathrm{C}$ and $\mathrm{Va}$ mixing on the interstitial sites. In BCC the size of interstitial sites is much smaller but there are 3 times as many and the CEF model is $(\mathrm{Fe})_{1}(\mathrm{C}, \mathrm{Va})_{3}$. The phase diagram for $\mathrm{C}-\mathrm{Fe}$ from an assessment by Ref 15 and the calculated Gibbs energy curves are shown in Fig. 3, the reference states are BCC for $\mathrm{Fe}$ and graphite for $\mathrm{C}$. Note the large extension of the metastable range of the Gibbs energies.

The chemical potential of $\mathrm{C}$ in $\mathrm{BCC}$ iron can be obtained by taking the difference between the partial Gibbs energy for two endmembers $G_{\mathrm{Fe}: \mathrm{Va}}$ and $G_{\mathrm{Fe}: \mathrm{C}}$, which at equilibrium are the sum of the chemical potentials of the components and $G_{\mathrm{Va}}=0$ :

$$
\begin{aligned}
G_{\mathrm{Fe}: \mathrm{Va}} & =G_{\mathrm{Fe}} \\
G_{\mathrm{Fe}: \mathrm{C}} & =G_{\mathrm{Fe}}+3 G_{\mathrm{C}}
\end{aligned}
$$

where the first term represents the chemical potential of $\mathrm{Fe}$ (because Va has zero chemical potential at equilibrium). A simple subtraction gives the chemical potential of $\mathrm{C}$ : 


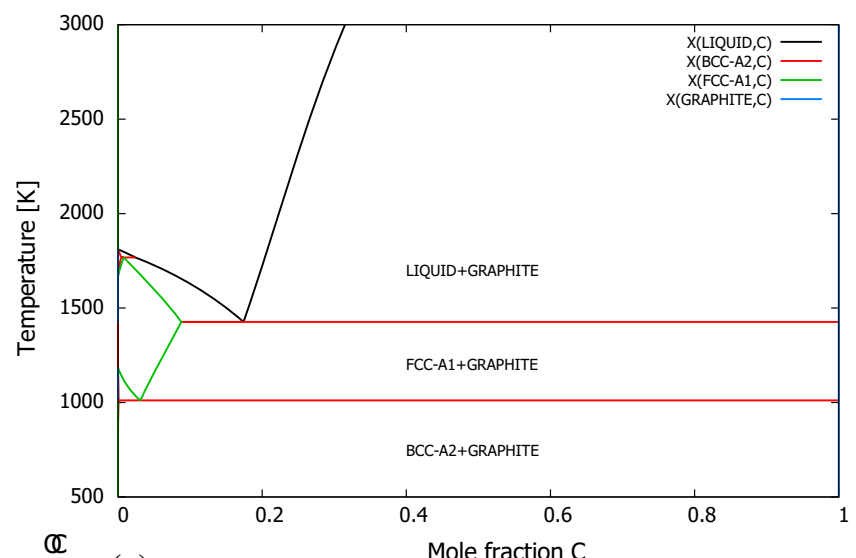

(a)

Fig. 3 The phase diagram for C-Fe in (a) has interstitial solution of C in the BCC and FCC phases. In (b) the Gibbs energy curves at $1000 \mathrm{~K}$ are shown. The curves for FCC and BCC ends at 50\% $\mathrm{C}$ and $75 \%$

$$
G_{\mathrm{C}}=\frac{1}{3}\left(G_{\mathrm{Fe}: \mathrm{C}}-G_{\mathrm{Fe}: \mathrm{Va}}\right)
$$

For some CEF based models it is not possible to extract the chemical potentials of each component.

In Fig. 4 the Gibbs energy surface for the FCC phase in the $\mathrm{C}-\mathrm{Fe}-\mathrm{Ti}$ system from an assessment by Ref 16 is shown modeled with 2 sublattices as $(\mathrm{Fe}, \mathrm{Ti})_{1}(\mathrm{C}, \mathrm{Va})_{1}$. The four corners represent pure FCC Fe, the (metastable) pure FCC $\mathrm{Ti}$, the stable cubic $\mathrm{TiC}$ carbide and the metastable cubic $\mathrm{FeC}$ carbide. The very low Gibbs energy for the cubic carbide in the $\mathrm{TiC}$ corner explains that this can be in equilibrium with the Fe-rich austenite.

The Gibbs energy difference between the diagonals of the constitutional square is:

$$
\Delta G_{\mathrm{CFeTi}}^{\mathrm{FCC}}={ }^{\circ} G_{\mathrm{Fe}: \mathrm{Va}}^{\mathrm{FCC}}+{ }^{\circ} G_{\mathrm{Ti}: \mathrm{C}}^{\mathrm{FCC}}-\left({ }^{\circ} G_{\mathrm{Fe}: \mathrm{C}}^{\mathrm{FCC}}+{ }^{\circ} G_{\mathrm{Ti}: \mathrm{Va}}^{\mathrm{FCC}}\right)
$$

where the endmember ${ }^{\circ} G_{\mathrm{Fe}: \mathrm{Va}}^{\mathrm{FCC}}$ represent the stable austenite and ${ }^{\circ} G_{\mathrm{Ti}: \mathrm{C}}^{\mathrm{FCC}}$ the stable cubic TiC carbide. ${ }^{\circ} G_{\mathrm{Fe}: \mathrm{C}}^{\mathrm{FCC}}$ is the Gibbs energy of a metastable cubic carbide for $\mathrm{Fe}$ and ${ }^{\circ} G_{\mathrm{Ti}: \mathrm{Va}}^{\mathrm{FCC}}$ is the Gibbs energy of a metastable FCC structure for Ti. Many metastable endmembers can today be calculated by DFT using some care. ${ }^{[17]}$

The reciprocal Gibbs energy has turned out to be an important parameter also for an approximation of the short range order (SRO) as described in section 3.2.

\subsubsection{Oxides and Other Compounds with Ionic Constituents}

Intermetallics, carbides, oxides ${ }^{[18]}$ and other phases with stoichiometric constraints of the components can be modeled with CEF. In the assessment of $\mathrm{Fe}-\mathrm{O}^{[19]}$ shown in Fig. 5(a) the wustite phase with a B1 structure was

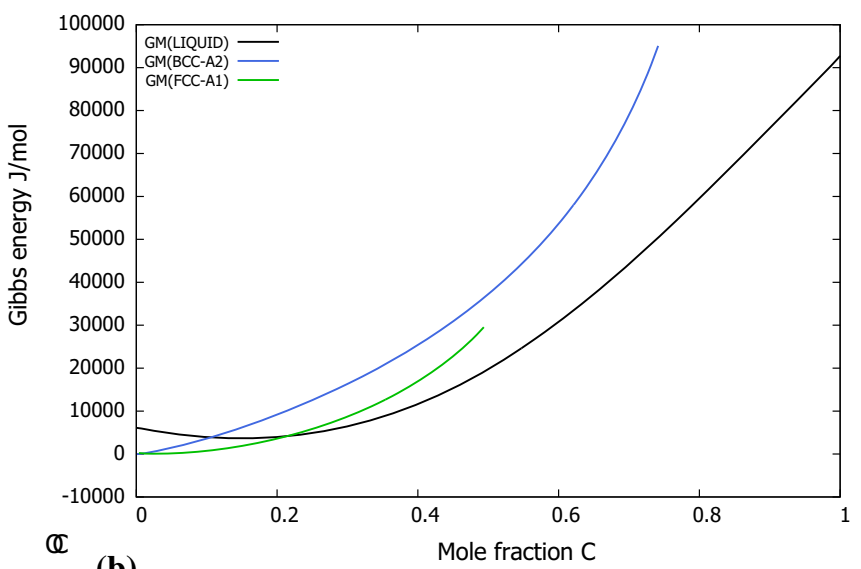

(b)

respectively as that is the maximum solubility of $\mathrm{C}$ according to the CEF model. The liquid phase is modeled as a regular solution and extends to pure $\mathrm{C}$

modeled using ions: $\left(\mathrm{Fe}^{+2}, \mathrm{Fe}^{+3}, \mathrm{Va}\right)_{1}\left(\mathrm{O}^{-2}\right)_{1}$, where the vacancies are needed to maintain electroneutrality and the magnetite with a spinel structure with $\mathrm{O}$ forming an FCC lattice with $\mathrm{Fe}$ ions in both the tetrahedral and octahedral interstitial sites: $\left(\mathrm{Fe}^{+2}, \mathrm{Fe}^{+3}\right)_{1}\left(\mathrm{Fe}^{+2}, \mathrm{Fe}^{+3}, \mathrm{Va}\right)_{2}\left(\mathrm{O}^{-2}\right)_{4}$. This model can describe both the composition range and the fact that magnetite is an inverse spinel with mainly $\mathrm{Fe}^{+3}$ on the tetrahedral sites and equal amount of $\mathrm{Fe}^{+2}$ and $\mathrm{Fe}^{+3}$ on the octahedral sites.

In the U-O phase diagram in Fig. 5(b) the $\mathrm{UO}_{2}$ phase has a $\mathrm{C} 1$ structure and its extensive composition range was modeled by Ref 20 with several valences of $U$ and with vacancies on the octahedral oxygen sites and interstitial $\mathrm{O}$ on the tetrahedral sublattice: $\left(\mathrm{U}^{+3}, \mathrm{U}^{+4}, \mathrm{U}^{+5}\right)_{1}\left(\mathrm{O}^{-2}, \mathrm{Va}\right)_{2}$ $\left(\mathrm{O}^{-2}, \mathrm{Va}\right)_{1}$.

In a quasibinary system like $\mathrm{Al}_{2} \mathrm{O}_{3}-\mathrm{MgO}$ assessed by Ref 21 shown in Fig. 5(c) there is also a spinel phase modeled as $\left(\mathrm{Al}^{+3}, \mathrm{Mg}^{+2}\right)_{1}\left(\mathrm{Al}^{+3}, \mathrm{Mg}^{+2}, \mathrm{Va}\right)_{2}\left(\mathrm{O}^{-2}\right)_{1}$ with a large composition range and there is also some solubility of $\mathrm{Al}$ in the Periclase phase. The model for the liquid phase in these systems is described using the two-sublattice ionic liquid model mentioned in section 4 and described in Ref 4.

\subsubsection{Modeling Defects in Ordered Phases}

Many intermediate phases in a system may have almost fixed composition and can in simpler cases be treated as such. However, in reality there exists always a small range of solubility and in some cases it is important to model this, for example in compound semiconductors. ${ }^{[22-26]}$

Defects can be vacancies, anti-site atoms, and interstitials and they can also be charged. When developing multicomponent databases the defects considered are 


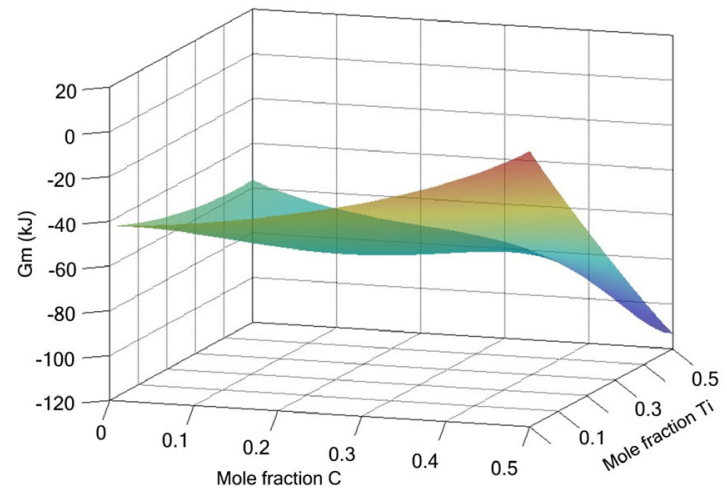

(a)

Fig. 4 A 3D surface of the Gibbs energy for the FCC phase in the FeTi-C system modeled with two sublattices in (a). It clearly shows that one can have an equilibrium between an Fe-rich FCC phase and the

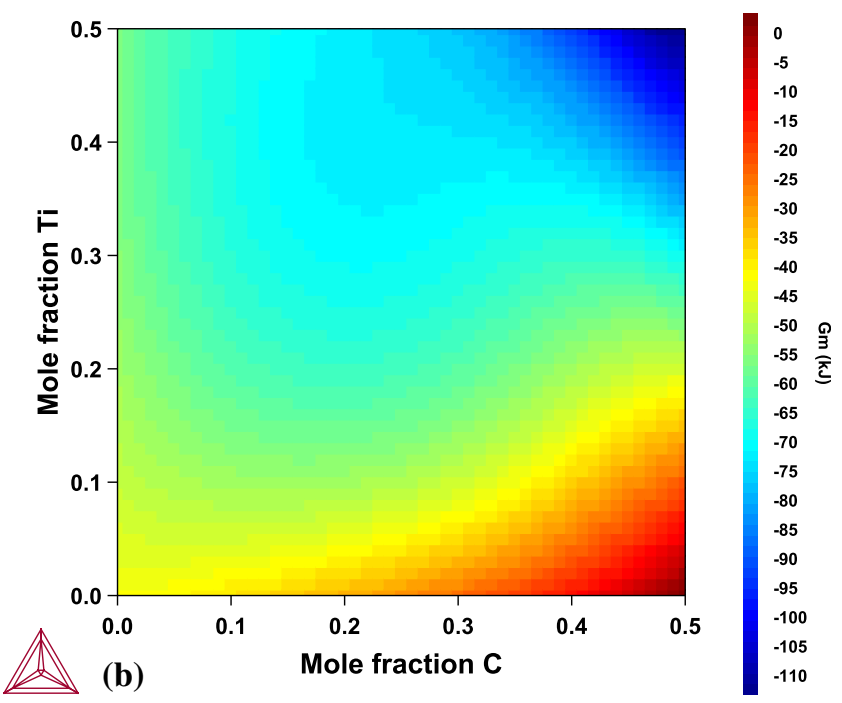

TiC phase with the same structure. In (b) the same surface from above with the colors indication the value of the Gibbs energy for varying compositions

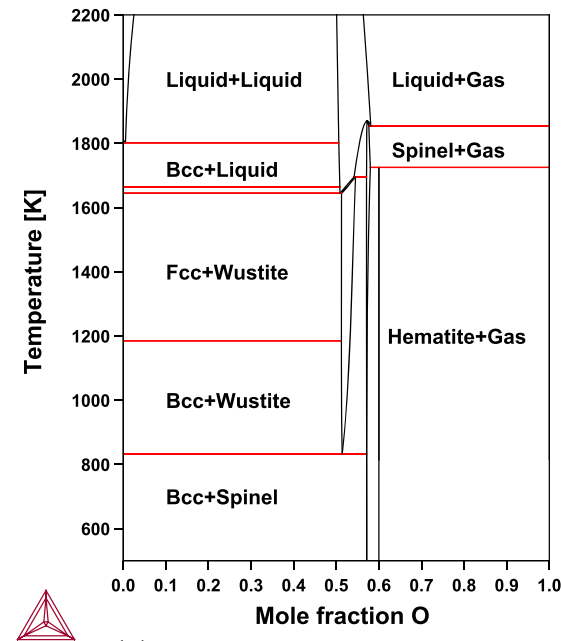

(a)

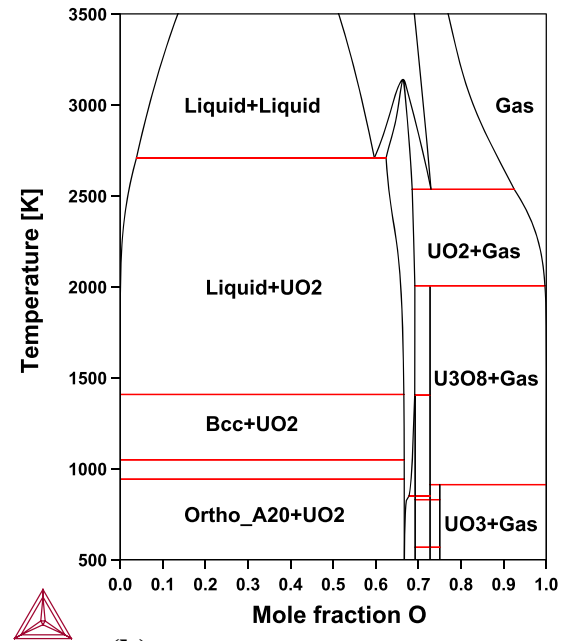

(b)

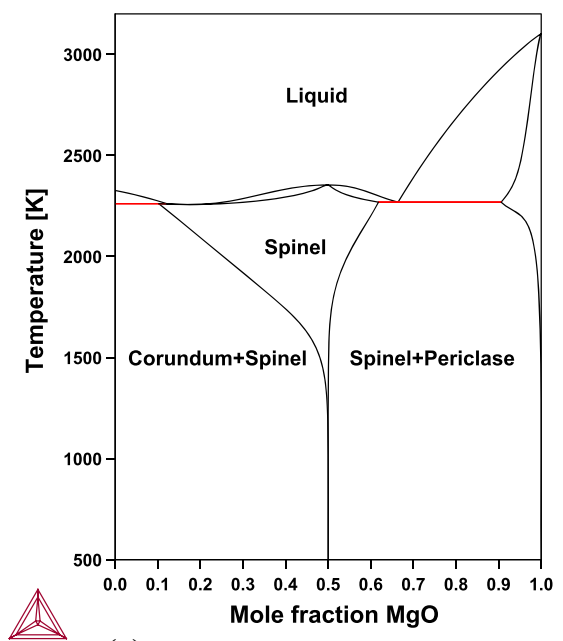

(c)
Fig. 5 Oxide system using CEF models with LRO for the solid phases. The phase diagram for $\mathrm{Fe}-\mathrm{O}$ in (a) has 3 oxides, wustite with B2 structure, magnetite and hematite with a corundum structure. In (b) the $\mathrm{O}-\mathrm{U}$ system with the high melting $\mathrm{UO}_{2}$ oxide with $\mathrm{C} 1$ structure which is used as fuel in most nuclear reactors. In (c) a

frequently simplified, for example the Laves phases, C14, $\mathrm{C} 15$ and $\mathrm{C} 36$ are generally modeled with anti-site atoms following a recommendation from the Ringberg workshop $^{[27]}$ even if there are cases in binary systems where other defects are dominant. The solubility range of an almost stoichiometric phase must not be ignored if the phase has a larger solubility in a ternary or higher order systems, for example the $\mathrm{B} 2$ phase in Fe-Ti extends to pure BCC-Ti inside the ternary Al-Fe-Ti system.

The frequently used Wagner-Schottky defect model ${ }^{[28]}$ uses 3 parameters, the Gibbs energy at the ideal quasibinary system $\mathrm{Al}_{2} \mathrm{O}_{3}-\mathrm{MgO}$ with a spinel with a wide solubility range and some solubility of $\mathrm{Al}$ in the periclase phase with a $\mathrm{B} 2$ structure. When assessing different binary systems it is important to use the same model for phases with the same structure because they may form solutions in higher order systems

composition and the increase of the Gibbs energy when adding a defect on either side. As described in Ref 4 this model can be implemented as a CEF model by introducing one more parameter representing the metastable phase consisting of only defects. In fact this has turned out to be a useful feature when developing databases because what is a defect in one binary system may be a normal constituent in another. A detailed discussion of models for defects can be found in Ref 12. 


\subsubsection{The Partitioned Model}

Modeling phases, such as TCP phases, with many sublattices and constituents leads to a large number of endmembers. A $\sigma$ phase with 5 sublattices in a six component system allowing all components to enter all sublattices has $6^{5}=7776$ endmembers. That would be very cumbersome to use in a simulation and in addition it is practically impossible to obtain the Gibbs energies of all these endmembers even using DFT. In fact almost all of these endmembers represent metastable or even unstable compounds (see section 5) and can safely be set to zero without affecting the stable equilibria with this phase. Only those endmembers that represent configurations that are stable or close to be stable need an accurate endmember energy but it is not a trivial task to identify such endmembers.

These endmember energies together with a configuration independent excess Gibbs energy can be adjusted to describe the experimental information of the stability range of the TCP phase. The configurantion independent excess term depends only on the mole fractions which can be calculated from the sublattice constitutions as given by Eq 6. The Gibbs energy for this regular solution also includes the Gibbs energies of the pure elements in the TCP phase structure when all sites are occupied by the same element. The total Gibbs energy for the TCP phase includes all this with the configurational entropy calculated using sublattices:

$$
\begin{aligned}
G_{M}= & \sum_{i} x_{i}{ }^{\circ} G_{i}+\sum_{I \in J} \Pi_{I}\left(y_{i \in I_{s}}\right){ }^{\circ} G_{I} \\
& +R T \sum_{s} a_{s} \sum_{i} y_{i s} \ln \left(y_{i s}\right)+{ }^{E} G_{M}\left(x_{i}\right)
\end{aligned}
$$

where the value of ${ }^{\circ} G_{i}$ is relative to a defined reference state for element $i$ and the endmember energies, ${ }^{\circ} G_{I}$, are relative to ${ }^{\circ} G_{i}$ for the same phase. The endmembers $I$ are the subset of all possible endmembers $J$ with non-zero value of ${ }^{\circ} G_{I}$. The excess Gibbs energy ${ }^{E} G_{M}\left(x_{i}\right)$ is calculated using the mole fractions. This very useful combination of a sublattice configurational entropy and regular solution excess Gibbs energy was first proposed by $\operatorname{Dupin}^{[29]}$ and later published ${ }^{[30]}$ and has been used to model several alloy systems shown in Fig. 6.

\subsubsection{TCP Phases and Similar}

The method to partition the Gibbs energy into a configuration dependent and a configuration independent excess energy explained in the previous section is particularly useful describing TCP phases like $\sigma, \chi$ and $\mu$ phases. These have all a slightly distorted BCC lattice with several sublattices and most elements can enter any sublattices but some are preferred because of different atomic sizes and bonds. In Fig. 7 the Gibbs energy curves for several phases in the Mo-Re system at $2000 \mathrm{~K}$ are shown across the composition range together with the constitutions of the $\sigma$ and $\chi$ phases modeled with 5 and 4 sublattices respectively.

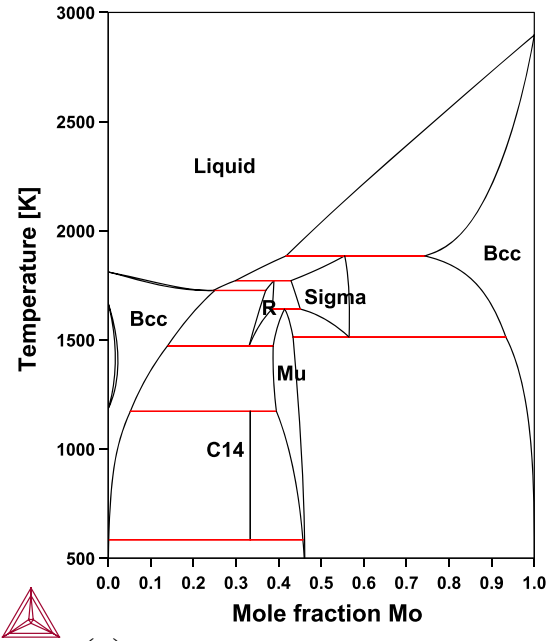

(a)

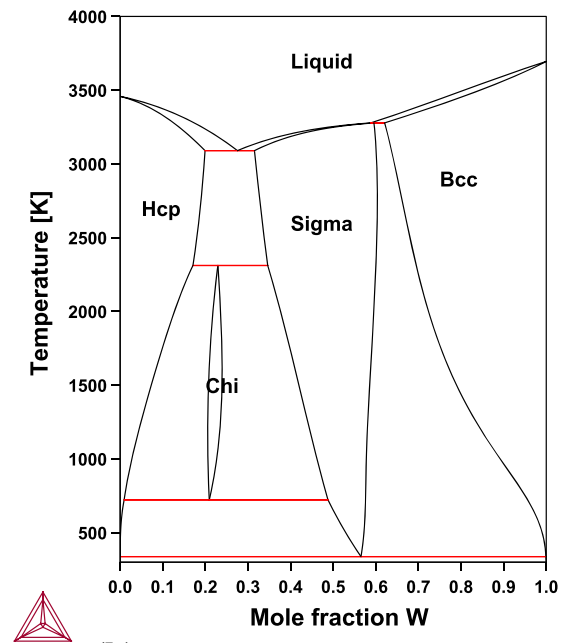

(b)

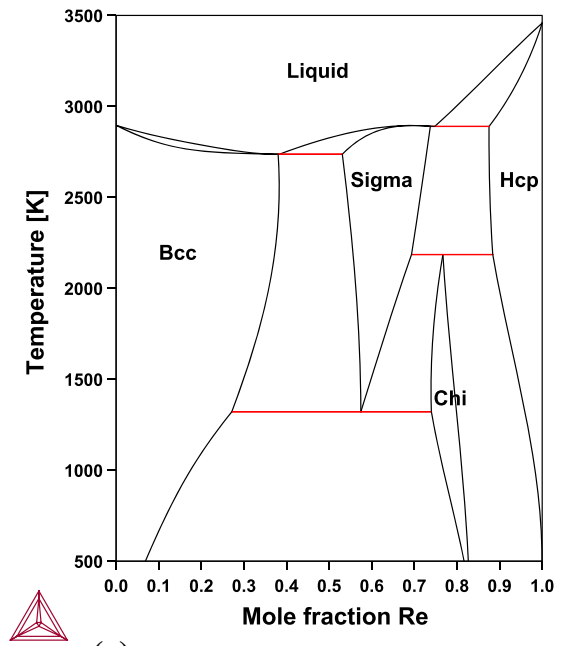

(c)
Fig. 6 The phase diagram for Fe-Mo by Ref 31 in (a) with the Laves, $\mu, \sigma$ and R phases. In (b) the Re-W phase diagram by Ref 32 with $\sigma$ and $\chi$ and in (c) the Mo-Re phase diagram by Ref 33 also with $\sigma$ and $\chi$ phases. These elements are important alloying addition in many alloys and as TCP phases are bad for the mechanical properties of the alloy, the stability range of the TCP phases must be carefully controlled in multicomponent alloys 


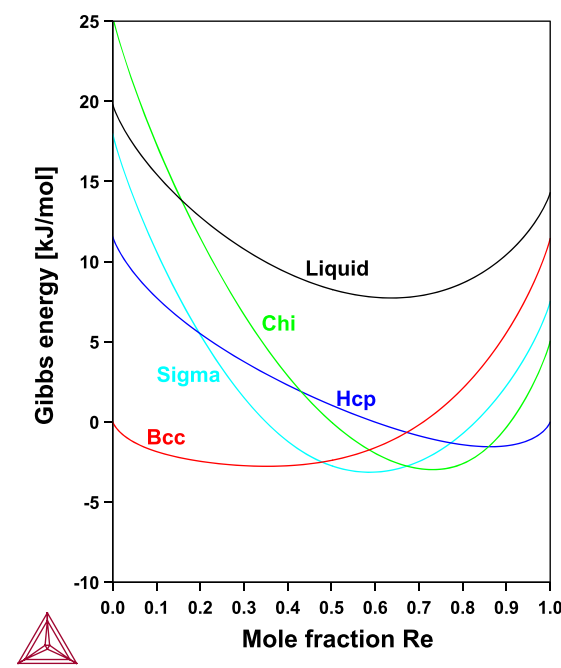

(a)

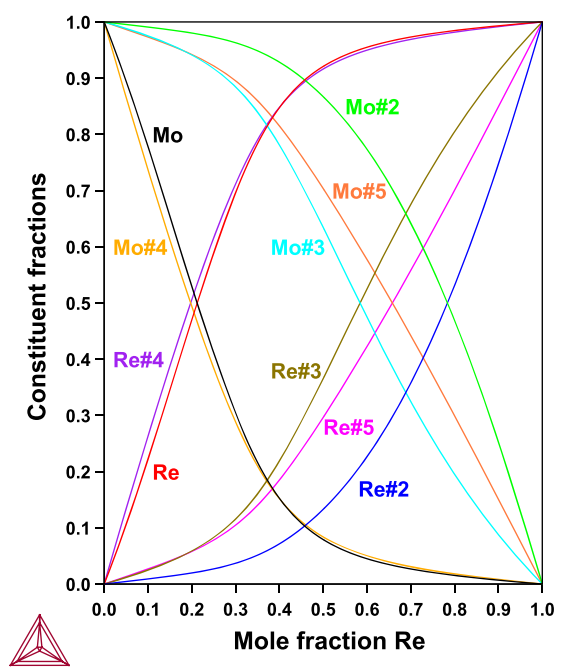

(b)

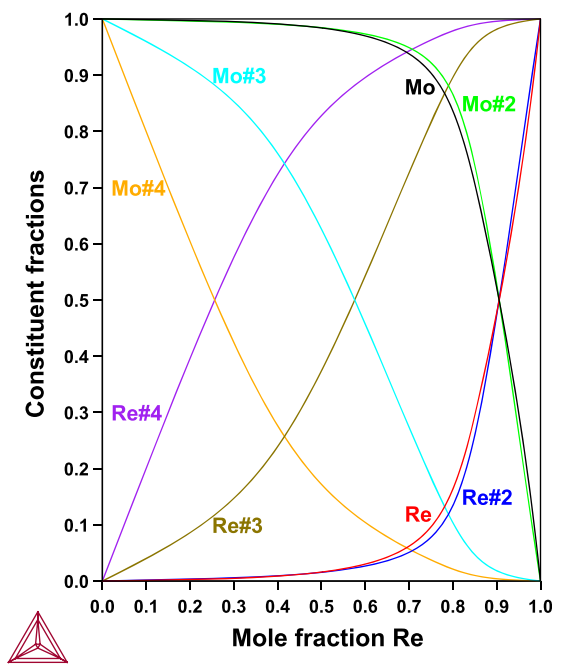

(c)
Fig. 7 The Gibbs energy curves at $1500 \mathrm{~K}$ for the Mo-Re system in (a) and the constitution of the $\sigma$ phase across the system in (b) and that of the $\chi$ phase in (c) showing which sublattices are preferred by the

In most commercial databases the TCP phases are modeled with fewer sublattices than required by the crystal structure and also with a reduced set of constituents on each sublattices. The reason for this is, as already mentioned, that a large number of endmembers makes assessments difficult and calculations slower.

But there are ways to improve the situation. Using the partitioned model we can describe the configurational entropy correctly and the excess parameters can be fitted with a regular solution of the components. The large number of possible endmembers can be reduced significantly by including only those which would lower the Gibbs energy of the phase. By intelligent guessing or maybe machine learning we can also reduce the number of DFT calculation necessary to determine those. Recently a very promising new way of describing the model parameters in TCP phases using effective bond energies has been proposed by Dupin et al. ${ }^{[34]}$

\subsubsection{SRO for Phases with LRO}

The effect of SRO is most important when the phase has no LRO. In fact it is not easy to separate the effect of SRO when there is also LRO and thus SRO can be modeled as a part of the LRO contribution. We should also consider that in Calphad modeling the origin of different physical phenomena that contribute to the Gibbs energy is frequently ignored unless it is very large and has a particular composition dependence like the ferromagnetic transition. different elements. Only a small composition range can be determined experimentally

\subsection{Phases with Order/Disorder Transition}

The technically most important phase with an order/disorder transition is without doubt the $\mathrm{L}_{2}$ ordered form of the FCC phase. This phase exists in the Al-Ni system around 25 mole\% of $\mathrm{Al}$ as shown in Fig. 8(b). The $\mathrm{L}_{2}$ phase is of central interest in Ni-based superalloys used for the turbine blades in most airplane engines due to its good corrosion and mechanical properties both at high and low $T$. There is intensive research ongoing to develop better and cheaper alloys based on this or similar ordered phases and calculations based on Calphad databases are used to reduce the experimental efforts because small variations in composition and heat treatments have significant effects. An additional problem is that many alloying additions may form TCP phases so careful composition and process control is needed.

\subsubsection{The Two-Sublattice Model for Order/Disorder}

The two-sublattice model for phases with order/disorder transformation has been used for long time developing commercial databases for Ni-based superalloys starting from the assessment of Al-Ni by Ansara et al. ${ }^{[35]}$ In the B2 ordered $\mathrm{BCC}$ phase in the $\mathrm{Al}-\mathrm{Ni}$ system the $\mathrm{Ni}$ atoms are replaced by vacancies on $\mathrm{Al}$ rich side. Thus the model used includes vacancies as constituent on all sublattices and this means one has to describe the properties of thermal vacancies in the BCC phase, this problem has been discussed recently in Ref 12 . 


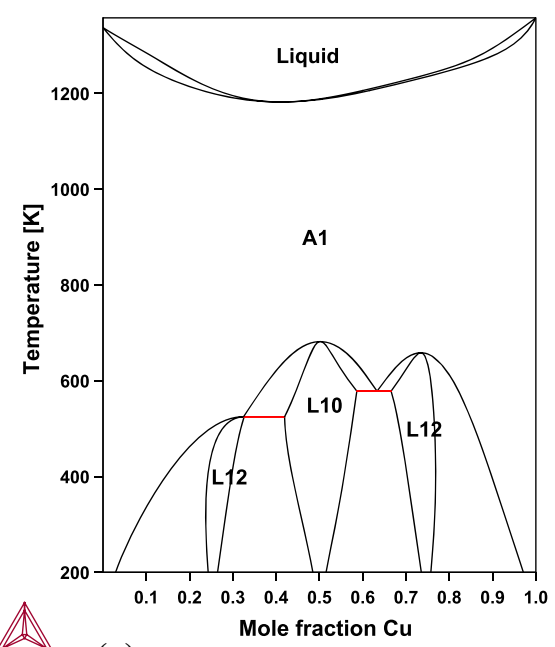

(a)

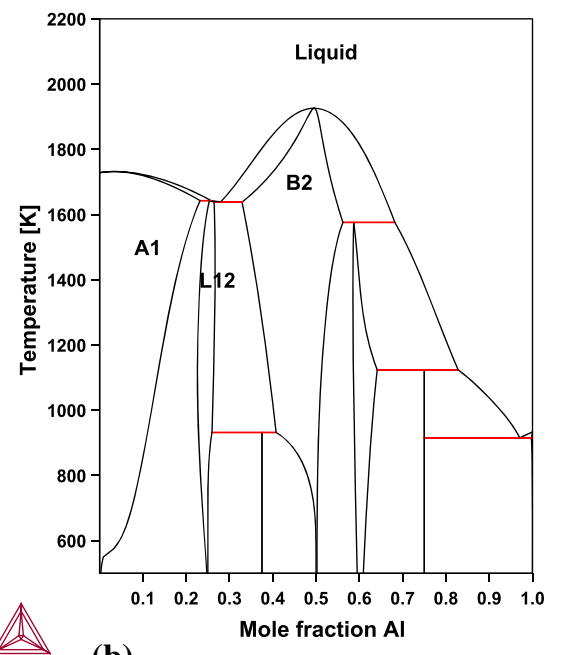

(b)

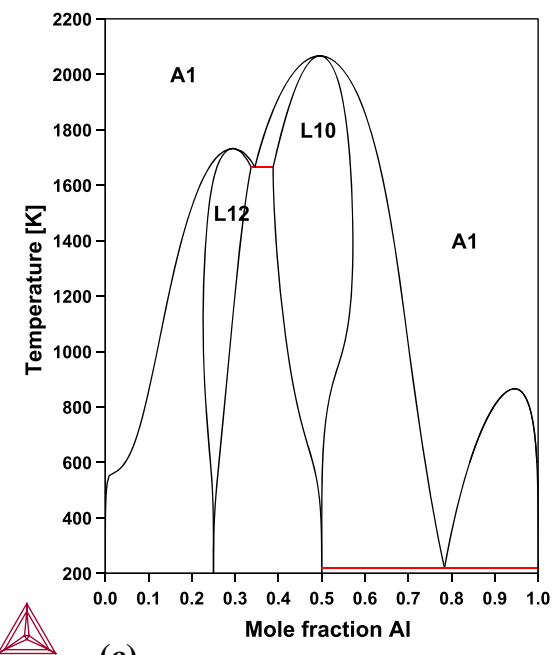

(c)
Fig. 8 The phase diagram for $\mathrm{Au}-\mathrm{Cu}$ by Ref 36 in (a), for $\mathrm{Al}-\mathrm{Ni}$ by Ref 35 in (b) and in (c). The diagram in (c) is a metastable diagram for just the FCC phase in Al-Ni showing also the $\mathrm{L} 1_{0}$ ordering. The parameters for the $\mathrm{L} 1_{0}$ ordering is from DFT calculations and there is

For the FCC phase the two-sublattice model is not symmetrical as one of the sublattices contains nearest neighbors. This requires a rather complicated addition of binary and ternary excess parameters when extending to multicomponent systems in order to ensure that the disordered state is described correctly. Moreover, the two-sublattice model used for the $\mathrm{L}_{2}$ ordered phase in $\mathrm{Al}-\mathrm{Ni}$ based systems, cannot describe the $\mathrm{L} 1_{0}$ ordered phase which is also an ordered form of the FCC phase and is stable for example in Al-Ti which is also of technical interest.

There is an interest to use a 4 sublattice model in databases but this step has not been made due to the fact that the increase of the number of constituent fractions in the 4 sublattice model increases significantly the time to calculate equilibria in multicomponent systems.

\subsubsection{The 4 Sublattice Model for Order/Disorder}

The first attempt to use CEF to model both $\mathrm{L}_{2}$ and $\mathrm{L}_{0}$ ordering in FCC was made by Ref 36 in an assessment of the $\mathrm{Au}-\mathrm{Cu}$ system, shown in Fig. 8(a). There has also been assessments of Al-Ni by Sundman and Dupin ${ }^{[37]}$ shown in Fig. 8 using the 4 sublattice model including the SRO approximation discussed in section 3.2.

Thermodynamic assessments using the 4 sublattice model are quite limited but there have been a number of publications for the ordered/disordered fcc phases $\left(\mathrm{L}_{2}\right.$ and A1) ${ }^{[38-40]}$ and bcc phases (Bcc_B2 and Bcc_A2)..$^{[41,42]}$ The advantage of the 4 sub-lattice model for the description of order/disorder phase transitions is that several ordered phases can be described by a single Gibbs energy function. But there are no commercial database yet with this model. no stable $\mathrm{L}_{2}$ phase on the Al-rich side in accordance with the DFT calculations. The SRO is approximated by a reciprocal parameter according to Eq 18

\subsubsection{Order/Disorder Models Requiring More than 4 Sublattices}

The $\kappa$ phase in Al-Fe-C has a perovskite structure and has ordering both on the substitutional sites for $\mathrm{Al}$ and $\mathrm{Fe}$ and on the interstitial sublattice with $\mathrm{C}$ and $\mathrm{Va}$. This requires 8 sublattices to be modeled:

$$
\begin{gathered}
(\mathrm{Al}, \mathrm{Fe})_{0.25}(\mathrm{Al}, \mathrm{Fe})_{0.25}(\mathrm{Al}, \mathrm{Fe})_{0.25}(\mathrm{Al}, \mathrm{Fe})_{0.25} \\
(\mathrm{Va}, \mathrm{C})_{0.25}(\mathrm{Va}, \mathrm{C})_{0.25}(\mathrm{Va}, \mathrm{C})_{0.25}(\mathrm{Va}, \mathrm{C})_{0.25}
\end{gathered}
$$

where the first 4 sublattices represent the almost stable $\mathrm{L}_{2}$ and $L 1_{0}$ ordering of the FCC phase in Al-Fe system and the last 4 an ordering of $\mathrm{C}$ and $\mathrm{Va}$ on the interstitial sublattices. But in the assessment by Connetable et al. ${ }^{[43]}$ the interstitial ordering was simplified by using only a quarter of the interstitial sites available in the disordered FCC.

Other cases when more than 4 sublattices would be needed is to model the $\mathrm{D}_{22}$ and $\mathrm{D} 0_{23}$ ordering of the FCC and HCP phases but as these phases have very little technological interest it is normally sufficient to treat them as separate phases, not as an ordered form of the FCC or HCP phases.

\subsubsection{The Partitioned Model for Phases with Order/ Disorder Transformations}

The partitioning of the Gibbs energy in an ordered and disordered part is possible also when the phase has an order/disorder transition but it has an importance difference in order to simplify the assessment of the ordering parameters. The reason is that phases like FCC, BCC and $\mathrm{HCP}$ exist as disordered in most system and the ordering is 
a feature that occurs only in a few systems. These systems normally also have the disordered phase stable in some composition and $T$ range. It is thus important to be able to assesses the parameters for the ordered and disordered parts independently. This is achieved by subtracting the Gibbs energy for the ordered part calculated after replacing the fractions in all sublattices with the disordered mole fractions.

$$
\begin{gathered}
G_{M}={ }^{\text {dis }} G_{M}+\Delta^{\text {od }} G_{M} \\
\Delta{ }^{\text {od }} G_{M}={ }^{\text {ord }} G_{M}(y)-{ }^{\text {ord }} G_{M}(y=x)
\end{gathered}
$$

where ${ }^{\mathrm{dis}} G_{M}$ is the Gibbs energy without the ordering sublattices and ${ }^{\text {ord }} G_{M}$ the Gibbs energy for the order/disorder using sublattices. The latter is calculated twice, once with the original sublattice fractions, $y$, and once with these replaced with the disordered fractions, $y=x$. If the phase is disordered we have $y=x$ and $\Delta^{\text {od }} G_{M}=0$.

This partitioning makes it easy to combine assessments where an FCC, BCC or HCP phase has an order/disorder phases with systems where the phase is always disordered because one just has to combine the parameters of the disordered parts.

The extra calculation of ${ }^{\text {ord }} G_{M}$ makes the use of this model slightly more complex but it is a necessary tool for those who assess and develop the thermodynamic databases. Note that the configurational entropy is included 3 times, in ${ }^{\text {dis }} G_{M}$, ${ }^{\text {ord }} G_{M}(y)$ and in ${ }^{\text {ord }} G_{M}(y=x)$ but the first and last will always cancel and do not have to be calculated.

\section{Short Range Ordering}

The CEF model for ordering has always been heavily criticized because it has no explicit SRO contribution. However, as shown in section 3.2 the CEF model has a term which provides an approximate contribution to SRO.

\subsection{CVM Based Models and MC Calculations}

The Cluster Variation Method (CVM) developed by Kikuchi $^{[3]}$ basically solved all problems modeling LRO and SRO together for phases with ordering. ${ }^{[44-50]}$ Kikuchi managed to derive an entropy expression using clusters of different sizes that gives the correct entropy expression. The criteria that the configuration is correct is that all clusters that include a specific lattice point must agree on the atom placed in that point.

However, when the model was published in 1951 there were no computers available that could cope with the complexity of the model. Not until $1976^{[44]}$ there was a computer software which made this model of practical interest. Even today with very fast computers CVM is very difficult to use for multicomponent systems and alternative methods like Monte-Carlo calculations ${ }^{[51-54]}$ are sometimes preferred. In both methods it can be of critical importance to select the correct set of clusters for each alloy system and this makes it difficult to combine assessments to create databases.

\subsubsection{CVM Tetrahedron Model for FCC}

For an FCC lattice using only tetrahedron clusters the model is simple with the configurational entropy:

$$
\begin{aligned}
{ }^{\mathrm{srf}} G_{M}^{\mathrm{CVM}-\mathrm{teta}} & =\sum_{i j k l} q_{i j k l}{ }^{\circ} G_{i j k l} \\
{ }^{\mathrm{cfg}} S_{M}^{\mathrm{CVM}-\mathrm{tetra}} & =-2 R\left(\sum_{i j k l} q_{i j k l} \ln \left(q_{i j k l}\right)\right. \\
& -\sum_{s t} \sum_{i j} p_{i s, j t} \ln \left(p_{i s, j t}\right) \\
& \left.+\frac{5}{4} \sum_{s} \sum_{i} y_{i s} \ln \left(y_{i s}\right)\right)
\end{aligned}
$$

where $q_{i j k l}$ are fractions of the tetrahedra clusters containing 4 atoms, each on a separate sublattice. There are 6 pairs in these tetrahedra and $p_{i s, j t}$ is the probability of an $i j$ pair between sublattice $s$ and $t$. Finally $y_{i s}$ the "point" probability (constituent fraction) of constituent $i$ on sublattice $s$ which can be different on each sublattice if there is LRO. The values of $p_{i s, j t}$ and $y_{i s}$ are calculated from the set of cluster fractions, $q_{i j k l}$. The indices $i, j, k, l$ represent the constituents on the 4 sublattices needed for the tetrahedra so the CVM model includes both LRO and SRO. If there is no LRO the point fractions on all sublattices are the same but as $q_{i j k l} \neq y_{i, 1} y_{j, 2} y_{k, 3} y_{l, 4}$ the entropy expression Eq 14 will still give an SRO contribution. Due to crystallograpic symmetry we have also several relations like:

$$
{ }^{\circ} G_{i j j j}={ }^{\circ} G_{j i j j}={ }^{\circ} G_{j j i j}={ }^{\circ} G_{j j j i}
$$

because all sites must be equivalent when the phase is disordered.

As already mentioned the CVM model requires a large number of clusters in a multicomponent system, a 4 sublattice FCC with 5 components using the tetrahedron model has $5^{4}=625$ clusters whereas a CEF model would have $5 \cdot 4=20$ constituent fractions and this gives a considerable computational advantage for the CEF model. A CVM based model certainly provides a better estimate SRO contribution than CEF in binary and ternary systems but in multicomponent system the number of clusters increases drastically and that means the contribution from each cluster to the SRO in the disordered state will decrease and the difference with a CEF model will also decrease. 
For the Shockley model the $\mathrm{L}_{2}$ and $\mathrm{L}_{0}$ ordered structures all disordered at the equiatomic composition as shown in Fig. 9(a). Furthermore, this transformation was of second order with no heat of transformation whereas experimentally there is a heat of transformation.

Adding the reciprocal parameters the CEF model we can obtain almost the same phase diagram as the CVM tetrahedron model and there is also a similar heat effect as shown in Fig. 10(a) for the equiatomic composition. The main difference between the CEF and CVM models is in the heat capacity because the CEF model, using a constant for the reciprocal parameter, has no heat capacity due to SRO in the disordered state as shown in Fig. 10.

\subsubsection{The Quasichemical CVM Approximation}

As it is of interest for modeling SRO in liquids we explain the simplest CVM based model, i.e. the quasichemical model assuming two sublattices with $z$ bonds between the sublattices and with the restriction that all nearest neighbor

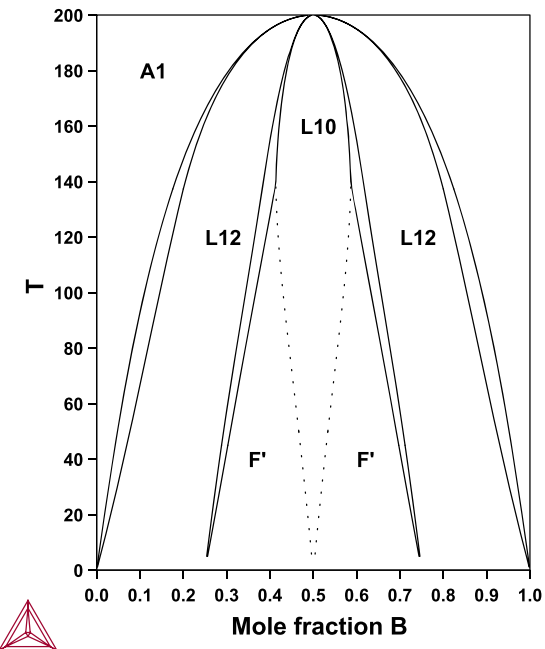

(a)

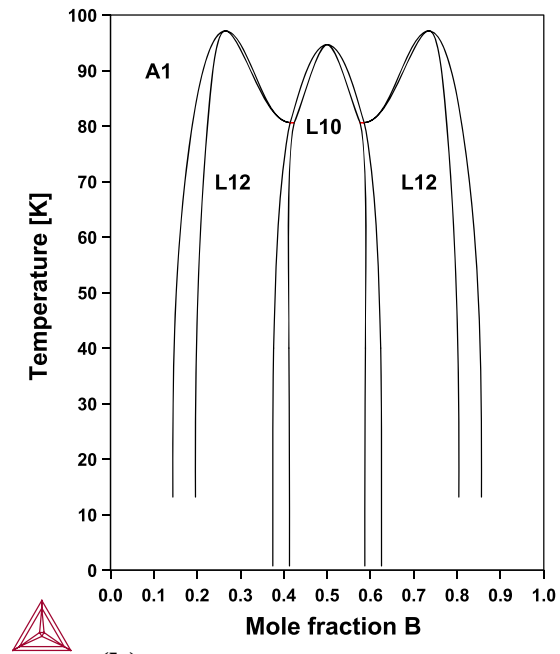

(b)

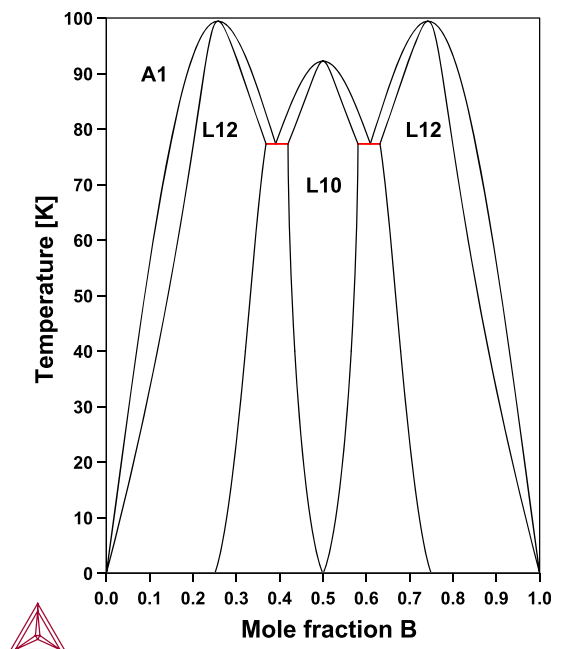

(c)
Fig. 9 The phase diagram calculated by Shockley in 1938 using a single bond energy for the A-B bond and the Bragg-Williams model for the entropy in (a). In (b) the CVM tetrahedron model using the same bond energy but the CVM tetrahedron configurational entropy. In (c) the CEF model using the same bond energy and Bragg-
Williams configurational entropy and a reciprocal parameter according to Eq 18. All diagrams are calculated with the same single bond energy. Note the temperature axis in (a) extends to the double value of (b) and (c)

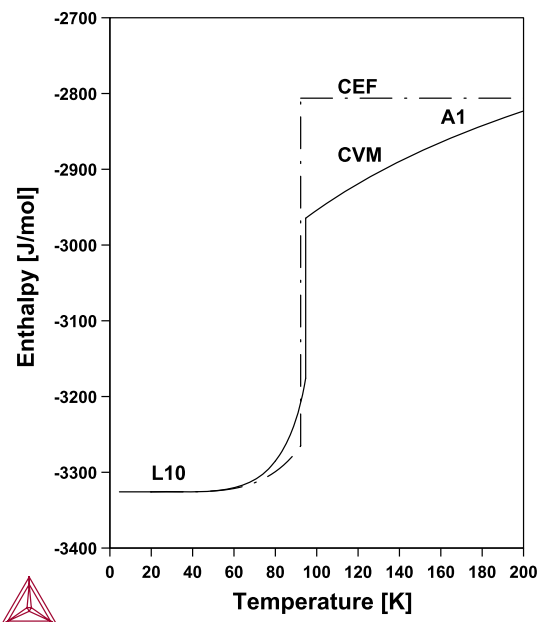

(a)

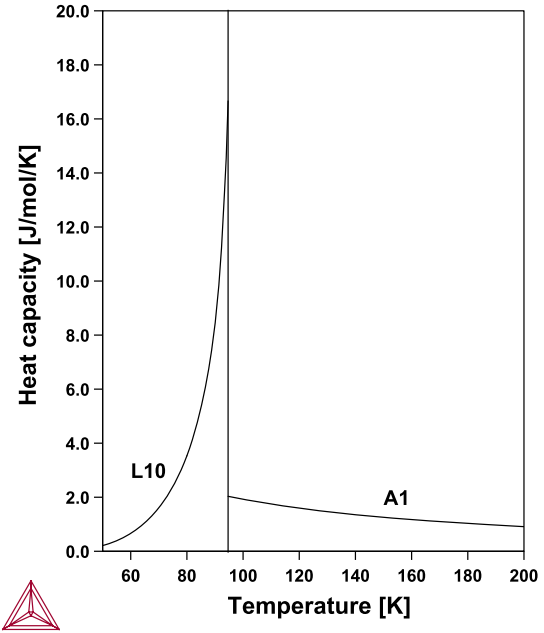

(b)

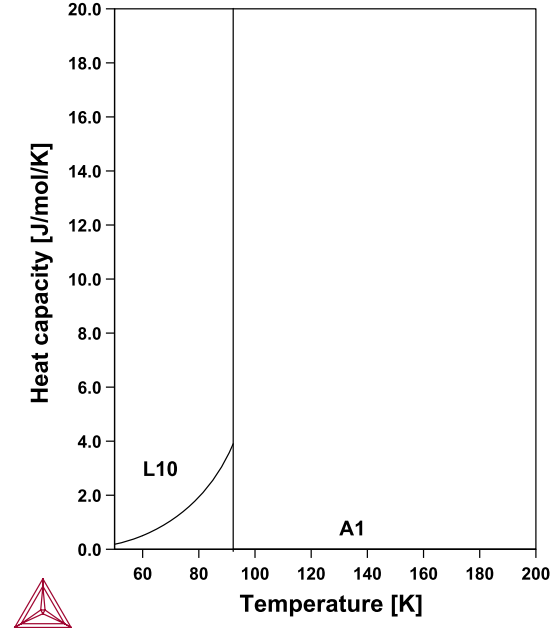

(c)

Fig. 10 In (a) the heat of transformation from $\mathrm{L} 1_{0}$ to $\mathrm{A} 1$ is shown for the CVM and CEF models. In (b) the heat capacity for the CVM tetrahedron model is shown in the ordered and disordered state and there are SRO ordering that gradualy decrease. In (c) the heat capacity

in the ordered state is due to the gradual disordering on the sublattices but there is no heat capacity effect due to SRO in the disordered state using a temperature independent reciprocal parameter in $\mathrm{Eq} 18$ 
bonds are with the other sublattice. Such restrictions are possible for the simple cubic (SC) and the BCC lattice but not for FCC. The two terms in the Gibbs energy expression for this model are:

$$
\begin{aligned}
{ }^{\mathrm{srf}} G_{m} & =\sum_{i} \sum_{j} p_{i j}{ }^{\circ} G_{i j} \\
{ }^{\mathrm{cfg}} S_{m} & =-\frac{z R}{2} \sum_{i} \sum_{j} p_{i j} \ln \left(\frac{p_{i j}}{y_{i}^{\prime} y_{j}^{\prime \prime}}\right) \\
& -R\left(\sum_{i} y_{i}^{\prime} \ln \left(y_{i}^{\prime}\right)+\sum_{i} y_{i}^{\prime \prime} \ln \left(y_{i}^{\prime \prime}\right)\right)
\end{aligned}
$$

where $p_{i j}$ is the probability of a pair between $i$ on first sublattice and $j$ on the second. $z$ is the number of nearest neighbours, for the SC that is 6 and for the $\mathrm{BCC}$ it is 8 . The FCC phase cannot be modeled with the quasichemical model as some nearest neighbours (pairs) would share the same sublattice, FCC ordering requires at least the CVM tetrahedron model with 4 sublattices.

Note that when there is LRO we have $p_{i j} \neq p_{j i}$ whereas when there is no LRO they are equal. The SRO is related to the difference:

$\epsilon_{i j}=p_{i j}-y_{i}^{\prime} y_{j}^{\prime \prime}$

and when there is no SRO $\epsilon_{i j}=0$ and $p_{i j}=y_{i}^{\prime} y_{j}^{\prime \prime}$ and we have an ideal configurational entropy because the first term in Eq 16 will vanish.

\subsubsection{The Cluster Site Approximation}

The quasi-chemical model ${ }^{[55]}$ for the non-random distribution of the pairs of neighboring sites was generalized by Yang and $\mathrm{Li}^{[56-58]}$ in 1940's to consider groups or clusters containing larger number of sites. Their assumption of the noninterference of the clusters gave an entropy equation involving only the cluster entropy and the single-site entropy. Due to this nature of Yang and Li's generalized quasi-chemical model, a new name, Cluster Site Approximation (CSA), was coined by Oates and Wenzl ${ }^{[59]}$ for highlighting the essence of the model. The advantage of the CSA model over the cluster variation method (CVM) is that the independent variables in the Gibbs energy functional are the site probabilities instead of the cluster probabilities. The use of only site probabilities in CSA reduces drastically the number of independent variables in the Gibbs energy functional. The number of equations that needs to be solved in the CSA model is in the order of $C \cdot n$ ( $C$ being the number of components and $n$ being the size of the cluster) instead of the $C^{n}$ required in CVM in which the cluster probabilities are used. Unlike the zeroth approximation, which is often referred to as Bragg-Williams approximation, however, the CSA model takes the short- range order into consideration, which is an important feature describing phases that undergo an order/disorder transition.

In order to improve the accuracy of the original CSA model, the number of energetically non-interfering clusters per site was suggested to be allowed to act as an adjustable parameter that can be fitted to either Monte Carlo (MC) simulation results or experimental data. ${ }^{[60-62]}$ Zhang et al. ${ }^{[61]}$ demonstrated that using the relaxed CSA model they can obtain an improved thermodynamic description of the fcc order/disorder transition in the $\mathrm{Ni}-\mathrm{Al}$ system with the use of fewer model parameters than previous descriptions using CEF. To the best of our knowledge, so far the CSA model has been employed to model the $\mathrm{Au}-\mathrm{Ni}, \mathrm{Au}-\mathrm{Cu}, \mathrm{Au}-\mathrm{Ni}, \mathrm{Cd}-\mathrm{Mg},{ }^{[63]} \mathrm{Cr}^{-\mathrm{Pt}^{[64]}}$ and $\mathrm{Cr}-$ $\mathrm{Ir}^{[65]}$ binary systems. The successful applications of CSA approximation to fcc phases $\left(\mathrm{Ll}_{2}\right.$ and $\left.\mathrm{A} 1\right)$ in a few ternary systems ${ }^{[62,}{ }^{66-68]}$ and even two quaternary systems of $\mathrm{Ni}$ $\mathrm{Al}-\mathrm{Cr}-\mathrm{Re}^{[69]}$ and Ni-Al-Cr-Ir ${ }^{[70]}$ have been demonstrated, but so far no multicomponent database has been built using this model.

\subsection{The SRO Approximation in CEF Using Reciprocal Parameters}

In 1998 Sundman et al. ${ }^{[36]}$ derived an expression for the contribution to the Gibbs energy due to SRO for a quasichemical CVM based model. They found that the first term of a Taylor expansion of the SRO contribution according to Eq 16 to the Gibbs energy is:

$-y_{\mathrm{A}}^{\prime} y_{\mathrm{B}}^{\prime} y_{\mathrm{A}}^{\prime \prime} y_{\mathrm{B}}^{\prime \prime} \frac{\left(\Delta G_{\mathrm{AB}}\right)^{2}}{z R T}$

where $y$ represent the constituent fractions (point probabilities) on the different sublattices, $z$ the number of bonds between the sublattices and $\Delta G_{\mathrm{AB}}$ is the so called reciprocal Gibbs energy, already mentioned in section 2.3.1, for the difference of the diagonals of the endmembers of the model:

$$
\Delta G_{\mathrm{AB}}={ }^{\circ} G_{\mathrm{A}: \mathrm{A}}+{ }^{\circ} G_{\mathrm{B}: \mathrm{B}}-{ }^{\circ} G_{\mathrm{A}: \mathrm{B}}-{ }^{\circ} G_{\mathrm{B}: \mathrm{A}}
$$

In a CEF model such a parameter is denoted $L_{\mathrm{A}, \mathrm{B}: \mathrm{A}, \mathrm{B}}$ and referred to as a reciprocal parameter. As the term is negative and $\Delta G$ is squared it will always decrease the Gibbs energy.

This term is entropic as it is divided by $T$ but in most cases the $L_{\mathrm{A}, \mathrm{B}: \mathrm{A}, \mathrm{B}}$ parameter used to represent the SRO contribution has been taken as constant because extrapolations to low $T$ give wrong results with a $T^{-1}$ term. In practice the SRO contribution is most important at the temperature of the order/disorder transition and above and the reciprocal parameter is adjusted to fit this. That means 
the SRO contribution will normally not decrease with $T$ and there is no contribution to the heat capacity when the phase is disordered. When calculating Fig. 9(c) and 10(c) the reciprocal parameter was set equal to the bond energy used in the ${ }^{\circ} G_{i j k l}$ parameters.

Note that due to the partitioning of the Gibbs energy for phases with order/disorder transformations the reciprocal parameters must be included in the disordered part as an excess Gibbs energy because SRO is most important when the phase is disordered. How to do this is explained in Lukas et al. ${ }^{[4]}$

All the calculations with the CEF model for systems with order/disorder transitions, as in Fig. 2(a), have this SRO contribution, except where specifically mentioned. More examples adopting this approach can be found in Ref 42, 71, and 72 .

\subsection{SRO as Entropy and/or Enthalpy Contribution}

SRO changes the local configuration to minimize the total energy of the system and this affects both the enthalpy and the configurational entropy. The reciprocal model does not introduce any explicit constituent variable to describe SRO and has thus no effect on the configurational entropy. However, the reciprocal parameter can depend on $T$ and thus contribute to the entropy as well as the enthalpy of the system.

\section{SRO in Liquids}

In liquids the atoms have no fixed positions but we may find very strong SRO at certain compositions.

The following types of liquid models are described in more detail by Ref 4:

The associate model reduces the configurational entropy due to SRO by introducing a molecule with the composition related to the composition of strong SRO. It is called the associate model because the associate is a modeling tool, there is no assumption that the molecule really exists. ${ }^{[3]}$ This model has problems in multicomponent systems as the interaction between the associates are difficult to determine and extrapolations are uncertain. At very dilute compositions the associates increase the configurational entropy and may give wrong extrapolations. The quasichemical model without LRO is used by the FactSage group in Montereal. It has been used successfully for multicomponent databases of oxide liquid and matte phases $^{[74-78]}$ and recently also for metallic liquids. It has no LRO transition and thus the bond probabilities $p_{i j}=p_{j i}$ always. The number of bonds, $z$ in $\mathrm{Eq} 16$ is set to a physically unrealistic value, $z=2$, but this avoids the problem with negative configurational entropy when the bond energy is so strong that liquid would like to have LRO, i.e. $p_{i j} \neq p_{j i}$. But it also means the model requires additional parameters to describe liquid miscibility gaps. There are several commercial databases using this model.

The corrected quasichemical model was proposed by Hillert et al. ${ }^{[79]}$ and use a realistic number of bonds in the liquid but it has never been implemented and tested for a real system.

The ionic two sublattice model was proposed by Hillert et al. ${ }^{[80]}$ This model treat SRO as LRO in the liquid based on the Temkin ${ }^{[10]}$ assumption that molten salts can be considered having two sublattices, not with fixed sites in space but a complete separation of cations and anions and that a cation never will take the place of an anion as that would require a large amount of energy. The Temkin model has been expanded to include also liquids without anions and with neutral constituents. An interesting fact is that the normal regular solution model, used for metals, is a subset of this model and so is some cases of the associate model also. This model is used in several commercial databases for alloys, oxides and other systems.

The cell model for metallurgical slags was proposed by Kapoor et al. ${ }^{[81]}$ and used at Acelor-Mittal. ${ }^{[82,83]}$

The central atoms model was developed by Lupis and Elliott $^{[84]}$ and generalized by Foo and Lupis ${ }^{[85]}$ for multicomponent solid solutions in steels. The model has found applications in both liquid metals and metallurgical slags ${ }^{[86,87]}$ as well as amorphous metal alloys ${ }^{[88]}$ with good results.

\section{Assessment of Model Parameters}

Developing thermodynamic databases is a long term project, most of the currently available commercial databases were started more than 20 years ago. At the start of a database development it is important to select the models to be used with extreme care because changing the model for a phase later means that most of the parameters for that phase must be modified which is a significant effort. This fact is also a problem for those interested in developing new models.

Model parameters can normally not be measured directly and even if they can, such as enthalpies of formations, they must be adjusted to fit many different kinds of experimental data such as enthalpies of mixing, heat capacities, chemical potentials and phase diagram data. By default large metastable ranges of the phases are also included in an assessment but there is also an interest to have realistic metastable extrapolations because during a simulation of a phase transformation many of the phases 
will have compositions outside the stable range. For example rapid quenching will normally result in metastable states and it is important to be able to predict which phases that may appear using a Calphad database.

Over the last 20 years DFT calculations have provided valuable information in particular for metastable endmembers but sometimes the results represent so called "mechanically unstable" endmembers (with imaginary phonon frequencies) with values that are meaningless. ${ }^{[17]}$

\section{Summary}

When modeling ordering in alloys with the Calphad method there will always be a trade off between physical realism and obtaining useful results with reasonable efforts and computational times. Some 30 years ago the early $a b$ initio calculations seemed to make the Calphad modeling superfluous but today many physicists are interested to use the Calphad technique because they have found that the results from their calculations and experimental work need the mean field technique provided by the Calphad models in order to yield practically useful results in multicomponent alloys. The development of Calphad models has also gained from this collaboration.

The development of materials and processes for new demanding applications requires a practical tool like Calphad for describing the thermodynamics of multicomponent system with many solution phases, in particular for metastable states, during simulations at varying $T, P$ and phase compositions.

Acknowledgments One of the authors (Bo Sundman) acknowledges the distinguished professor program released by the Ministry of Education of China and the State Administration of Foreign Experts Affairs of China.

\section{References}

1. W. Shockley. Theory of Order for the Copper Gold Alloy System. The Journal of Chemical Physics, 6(3):130-144, 1938.

2. W.L. Bragg and E.J. Williams. The Effect of Thermal Agitation on Atomic Arrangement in Alloys. Proceedings of the Royal Society of London. Series A, 145(855):699-730, 1934.

3. R. Kikuchi. A theory of Cooperative Phenomena. Physical Review, 81(6):988, 1951.

4. H.L. Lukas, S.G. Fries, and B. Sundman. Computational Thermodynamics: the Calphad Method, volume 131. Cambridge University Press, Cambridge, United Kingdom, 2007.

5. O. Redlich and A.T. Kister. Algebraic Representation of Thermodynamic Properties and the Classification of Solutions. Industrial \& Engineering Chemistry, 40(2):345-348, 1948.

6. B. Sundman and J. Ågren. A Regular Solution Model for Phases with Several Components and Sublattices, Suitable for Computer Applications. Journal of Physics and Chemistry of Solids, 42(4):297-301, 1981.
7. B. Sundman, I. Ohnuma, N. Dupin, U.R. Kattner, and S.G. Fries. An Assessment of the Entire Al-Fe System Including D03 Ordering. Acta Mater., 57(10):2896-2908, 2009.

8. C.E. Campbell, U.R. Kattner, Z.-K. Liu (2014) The Development of Phase-based Property Data Using the CALPHAD Method and Infrastructure Needs. Integr. Mater. Manuf. Innov.; 3:12

9. M. Hillert and L.-I. Staffansson. The Regular Solution Model for Stoichiometric Phases and Ionic Melts. Acta Chemica Scandinavica, 24:3618-3626, 1970.

10. M. Temkin. Mixtures of Fused Salts as Ionic Solutions. Acta Phys Chim, 20:411-420, 1945.

11. M. Hillert. The Compound Energy Formalism. Journal of Alloys and Compounds, 320(2):161-176, 2001.

12. J. Rogal, S.V. Divinski, M.W. Finnis, A. Glensk, J. Neugebauer, J.H. Perepezko, S. Schuwalow, M.H.F. Sluiter, B. Sundman (2014) Perspectives on Point Defect Thermodynamics. Phys Status Solidi B; 251:97-129

13. M. Hillert. Some Viewpoints on the Use of Computer for Calculating Phase Diagrams. Physica B, 103B:31-40, 1981.

14. B. Sundman, X.-G. Lu, and H. Ohtani. The Implementation of an Algorithm to Calculate Thermodynamic Equilibria for MultiComponent Systems with Non-ideal Phases in a Free Software. Computational Materials Science, 101:127-137, 2015.

15. P. Gustafson. Thermodynamic Evaluation of the $\mathrm{Fe}-\mathrm{C}$ system. Scandinavian Journal of Metallurgy, 14:259-267, 1985.

16. B.-J. Lee. Thermodynamic Assessment of the Fe-Nb-Ti-C-N system. Metallurgical and Materials Transactions A, 32A:2423-2439, 2001.

17. A. van de Walle, R. Sun, Q.-J. Hong, S. Kadkhodaei (2017) Software Tools for High-throughput Calphad from First-principles Data. Calphad; 58:70-81

18. T.I. Barry, A.T. Dinsdale, J.A. Gisby, B. Hallstedt, M. Hillert, B. Jansson, S. Jonsson, B. Sundman, and J.R. Taylor. The Compound Energy Model for Ionic Solutions with Applications to Solid Oxides. Journal of Phase Equilibria, 13(5):459-475, 1992.

19. B. Sundman. An Assessment of the Fe-O System. Journal of Phase Equilibria, 12:127-140, 1991.

20. C. Guéneau, M. Baichi, M.D. Labroche, C. Chatillon, B. Sundman. Thermodynamic Assessment of the Uranium-Oxygen System. J Nucl. Mater., 304:161-175, (2002).

21. B. Hallstedt. Thermodynamic Assessment of the System MgOA12O3. J Am. Ceram. Soc., 75(6):1497-1507, 1992.

22. I. Ansara, C. Chatillon, H.L. Lukas, T. Nishizawa, H. Ohtani, K. Ishida, M. Hillert, B. Sundman, B.B. Argent, A. Watson, T.G. Chart, Anderson T. A Binary Database for III-V Compound Semiconductor Systems. Calphad 18, 177-222 (1994)

23. Q. Chen, M. Hillert, B. Sundman, W.A. Oates, S.G. Fries, and R. Schmid-Fetzer. Phase Equilibria, Defect Chemistry and Semiconducting Properties of CdTe(s) - Thermodynamic Modeling. Journal of Electronic Materials, 27(8):961-971, 1998.

24. Q. Chen and M. Hillert. The Compound Energy Model for Compound Semiconductors. Journal of Alloys and Compounds, 245(1-2):125-131, 1996.

25. J.-B. Li and J.-C. Tedenac. Thermodynamic Modeling of Native Point Defects and Dopants of GaN Semiconductors. Journal of Electronic Materials, 31(4):321-326, 2002.

26. M.C. Peters, J.W. Doak, W.-W. Zhang, J.E. Saal, G.B. Olson, and P.W. Voorhees. Thermodynamic Modeling of the $\mathrm{PbX}(\mathrm{X}=\mathrm{S}, \mathrm{Te})$ Phase Diagram Using a Five Sub-lattice and Two Sub-lattice Model. Calphad, 58:17-24, 2017.

27. B. Sundman and F. Aldinger. Workshop on Thermodynamic Models and Data for Pure Elements and Other Endmembers of Solutions. Calphad, 19:437-571, 1995.

28. Wagner C. and W. Schottky. Theory of Arranged Mixed Phases. Z Phys. Chem. B, 11:163-210, 1930.

29. N. Dupin, Personal Communication. (1994) 
30. I. Ansara, B. Burton, Q. Chen, M. Hillert, A. FernandezGuillermet, S.G. Fries, H.L. Lukas, H.-J. Seifert, and W.A. Oates. Models for Composition Dependence. Calphad, 24(1):19-40, 2000.

31. J.-O. Andersson. A Thermodynamic Evaluation of the Fe-Mo-C System. Calphad, 12:9-23, 1988.

32. S.G. Fries and B. Sundman. Using Re-W Sigma-phase Firstprinciples Results in the Bragg-Williams Approximation to Calculate Finite-temperature Thermodynamic Properties. Phys. Rev. B 66, 012203 (2002)

33. R. Mathieu, N. Dupin, J.-C. Crivello, Y. Yaqoob, A. Breidi, J.-M. Fiorani, N. David, and J.-M. Joubert. Calphad Description of the Mo-Re System Focused on the Sigma Phase Modeling. Calphad, 43:18-31, 2013.

34. N. Dupin, B. Sundman, U.R. Kattner, S.G. Fries, M. Palumbo, Calphad meeting Mexico (2018)

35. I. Ansara, N. Dupin, H.L. Lukas, and B. Sundman. Thermodynamic Assessment of the Al-Ni System. Journal of Alloys and Compounds, 247:20-30, 1997.

36. B. Sundman, S.G. Fries, and W.A. Oates. A Thermodynamic Assessment of the Au-Cu System. Calphad, 22(3):335-354, 1998.

37. B. Sundman, N. Dupin, Thermodynamic Assessment of the Al-Ni System. JEEP Conference (2002)

38. X.-G. Lu, B. Sundman, and J. Ågren. Thermodynamic Assessments of the Ni-Pt and Al-Ni-Pt systems. Calphad, 33:450-456, 2009.

39. Y.-R. Wang, P. Zhou, Y.-B. Peng, Y. Du, B. Sundman, J.-Z. Long, T. Xu, and Z.-J. Zhang. A Thermodynamic Description of the Al-Co-Ni System and Site Occupancy in Co-AlNi3 Composite Binder Phase. J. Alloy Compd., 687:855-866, 2016.

40. B. Hu, X.-M. Yuan, Y. Du, J. Wang, and Z.-K. Liu. Thermodynamic Reassessment of the Ni-Si-Ti System Using a Four Sublattice Model for Ordered/Disordered fcc Phases Supported by First-principles Calculations. J. Alloy Compd., 693:344-56, 2017.

41. B. Hu, Y. Du, J.C. Schuster, W.-H. Sun, S.-H. Liu, and C.-Y. Tang. Thermodynamic Modeling of the Cr-Ni-Ti System Using a Four-sublattice Model for Ordered/Disordered bcc Phases. Thermochimica Acta, 578:35-42, 2014.

42. T. Abe and M. Shimono. A Description of the Effect of ShortRange Ordering in BCC Phases with Four Sublattices. Calphad, 45:40-48, 2014.

43. D. Connetable, J. Lacaze, P. Maugis, and B. Sundman. A Calphad Assessment of Al-C-Fe System with the $\kappa$ Carbide Modeled as an Ordered Form of the fcc Phase. Calphad, 32(2):361-370, 2008.

44. R. Kikuchi and D. de Fontaine. Calculation of $\mathrm{Cu}-\mathrm{Au}$ phasediagram by Cluster Variation Method. JOM, 28(12):A27, 1976.

45. J.M. Sanchez and D. de Fontaine. The fcc Ising Model in the Cluster Variation Approximation. Physical Review B, 17(7):2926, 1978.

46. J.M. Sanchez and D. de Fontaine. Ordering in fcc Lattices with First- and Second-Neighbor Interactions. Physical Review B, 21(1):216, 1980.

47. J.M. Sanchez and D. de Fontaine. Ising Model Phase Diagram Calculations in the fcc Lattice with First- and SecondNeighbor Interactions. Physical Review B, 25(3):1759, 1982.

48. J.M. Sanchez. Pair Correlations in the Cluster Variation Approximation. Physica A: Statistical Mechanics and its Applications, 111(1-2):200-216, 1982.

49. J.M. Sanchez, F. Ducastelle, and D. Gratias. Generalized Cluster Description of Multicomponent Systems. Physica A: Statistical Mechanics and its Applications, 128(1-2):334-350, 1984.

50. T. Mohri, J.M. Sanchez, and D. de Fontaine. Overview no. 43: Binary Ordering Prototype Phase Diagrams in the Cluster Variation Approximation. Acta Metallurgica, 33(7):1171-1185, 1985.
51. K. Binder, J.L. Lebowitz, M.K. Phani, and M.H. Kalos. Monte Carlo Study of the Phase Diagrams of Binary Alloys with Face Centered Cubic Lattice Structure. Acta Metallurgica, 29(9):1655-1665, 1981.

52. C. Bichara and G. Inden. Monte Carlo Calculation of the Phase Diagram of BCC Fe-Al Alloys. Scripta Metallurgica et Materialia, 25(11):2607-2611, 1991.

53. H. Ackermann, G. Inden, and R. Kikuchi. Tetrahedron Approximation of the Cluster Variation Method for bcc Alloys. Acta Metallurgica, 37(1):1-7, 1989.

54. A. van de Walle and M. Asta. Self-driven Lattice-Model Monte Carlo Simulations of Alloy Thermodynamic Properties and Phase Diagrams. Modelling and Simulation in Materials Science and Engineering, 10(5):521, 2002.

55. R.H. Fowler and E.A. Guggenheim. Statistical Thermodynamics. Cambridge University Press, Cambridge, United Kingdom, 1939.

56. C.N. Yang. A Generalization of the Quasi-Chemical Method in the Statistical Theory of Superlattices. The Journal of Chemical Physics, 13(2):66-76, 1945.

57. Y.-Y. Li. Quasi-Chemical Theory of Order for the Copper Gold Alloy System. The Journal of Chemical Physics, 17(5):447-454, 1949.

58. Y.-Y. Li. Quasi-Chemical Method in the Statistical Theory of Regular mixtures. Phys. Rev., 76(7):972-979, 1949.

59. W.A. Oates and H. Wenzl. The Cluster/Site Approximation for Multicomponent Solutions-a Practical Alternative to the Cluster Variation Method. Scr. Mater., 35(5):623-627, 1996.

60. W.A. Oates, F. Zhang, S.L. Chen, and Y.A. Chang. Improved Cluster-Site Approximation for the Entropy of Mixing in Multicomponent Solid Solutions. Physical Review B, 59(17):11221, 1999.

61. F. Zhang, Y.A. Chang, Y. Du, S.-L. Chen, and W.A. Oates. Application of the Cluster-Site Approximation (CSA) Model to the Fcc Phase in the Ni-Al System. Acta Materialia, 51(1):207-216, 2003.

62. W. Cao, J. Zhu, Y. Yang, F. Zhang, Chen S.-L., W.A. Oates, and Y.A. Chang. Application of the Cluster/Site Approximation to fcc Phases in Ni-Al-Cr System. Acta Mater. 53, 4189-4197 (2005)

63. J. Zhang, W.A. Oates, F. Zhang, S.-L. Chen, K.-C. Chou, and Y.A. Chang. Cluster/Site Approximation Calculation of the Ordering Phase Diagram for Cd-Mg Alloys. Intermetallics, 9:5-8, 2001.

64. C. Zhang, J. Zhu, A. Bengtson, D. Morgan, F. Zhang, Y. Yang, and Chang Y.A. Thermodynamic Modeling of the Cr-Pt Binary System Using the Cluster/Site Approximation Coupling with First-principles Energetics Calculation. Acta Mater., 56:5796-5803, 2008.

65. C. Zhang, J. Zhu, D. Morgan, Y. Yang, F. Zhang, W. Cao, and Chang Y.A. Thermodynamic Modeling of the Cr-Ir Binary System Using the Cluster/Site Approximation (CSA) Coupling with First-principles Energetic Calculation. Calphad, 33:420-424, 2009.

66. C. Zhang, J. Zhu, A. Bengtson, D. Morgan, F. Zhang, W.-S. Cao, and Y.A. Chang. Modeling of Phase Stability of the fcc Phases in the Ni-Ir-Al System Using the Cluster/Site Approximation Method Coupling with First-principles Calculations. Acta Mater., 56:2576-2584, 2008.

67. J. Zhu, C. Zhang, W. Cao, Y. Yang, F. Zhang, S.-L. Chen, D. Morgan, and Y.A. Chang. Experimental Investigation and Thermodynamic Modeling of the Ni-Al-Ru Ternary System. Acta Mater., 57:202-212, 2009.

68. J. Zhu, C. Zhang, D. Ballard, P. Martin, J. Fournelle, W. Cao, and Y.A. Chang. Study of the Ni-rich Multi-phase Equilibria in NiAl-Pt Alloys Using the Cluster/Site Approximation for the FaceCentered Cubic Phases. Acta Mater., 58:180-188, 2010. 
69. J. Zhu, W. Cao, Y. Yang, F. Zhang, S.-L. Chen, W.A. Oates, and Y.A. Chang. Application of the Cluster/Site Approximation to fcc Phases in the Ni-Al-Cr-Re System. Acta Mater., 55:4545-4551, 2007.

70. C. Zhang, F. Zhang, S.-L. Chen, W.S. Cao, and Y.A. Chang. Thermodynamic Modeling and Experimental Investigation of the Phase Stability at the Ni-rich Region of the Ni-Al-Cr-Ir System. Acta Mater., 57:6246-6256, 2011.

71. A. Kusoffsky. Thermodynamic Evaluation of the Ternary Ag-Au$\mathrm{Cu}$ System Including a Short Range Order Description. Acta Materialia, 50(20):5139-5145, 2002.

72. T. Abe and B. Sundman. A Description of the Effect of Short Range Ordering in the Compound Energy Formalism. Calphad, 27(4):403-408, 2003.

73. F. Sommer, Association Model for the Description of the Thermodynamic Functions of Liquid Alloys. I.-Basic Concepts. Zeitschrift Fur Metallkunde 73(2), 72-76 (1982)

74. M. Blander and A.D. Pelton. Thermodynamic Analysis of Binary Liquid Silicates and Prediction of Ternary Solution Properties by Modified Quasichemical Equations. Geochimica et Cosmochimica Acta, 51(1):85-95, 1987.

75. A.D. Pelton, S.A. Degterov, G. Eriksson, C. Robelin, and Y. Dessureault. The Modified Quasichemical Model I - Binary Solutions. Metallurgical and Materials Transactions B, 31(4):651-659, 2000.

76. A.D. Pelton and P. Chartrand. The Modified Quasi-Chemical Model: Part II. Multicomponent Solutions. Metallurgical and Materials Transactions A, 32(6):1355-1360, 2001.

77. A.D. Pelton, P. Chartrand, and G. Eriksson. The Modified QuasiChemical Model: Part IV. Two-sublattice Quadruplet Approximation. Metallurgical and Materials Transactions A, 32(6):1409-1416, 2001.

78. P. Chartrand and A.D. Pelton. The Modified Quasi-Chemical Model: Part III. Two Sublattices. Metallurgical and Materials Transactions A, 32(6):1397-1407, 2001.

79. M. Hillert, M. Selleby, and B. Sundman. An Attempt to Correct the Quasichemical Model. Acta Mater., 57(17):5237-5244, 2009.
80. M. Hillert, B. Jansson, B. Sundman, and J. Ågren. A Two-sublattice Model for Molten Solutions with Different Tendency for Ionization. Metallurgical Transactions A, 16(1):261-266, 1985.

81. M.L. Kapoor, M.G. Froberg, Theoretical Treatment of Activities in Silicate Melts. In Chemical Metallurgy of Iron and Steel: Proceedings of the International Symposium on Metallurgic Chemistry-Applications in Ferrous Metallurgy held in the University of Sheffield, 19th-21st July 1971, pages 17-22, Iron and Steel Institute, London (1973)

82. H. Gaye and J. Welfringer. Modelling of the Thermodynamic Properties of Complex Metallurgical Slags. In H.A. Fine and D.R. Gaskell, editors, Proc. Second International Symposium on Metallurgical Slags and Fluxes, pp 357-375, Warrendale, PA (1984).

83. H. Gaye, J. Lehmann, Modelling of Slag Thermodynamic Properties - From Oxides to Oxisulphides. In: Proceedings of the 5th International Conference on Molten Slags, Fluxes, and Salts '97: January 5-8, 1997, Sydney, Australia, pages 27-34, Iron and Steel Society, Warrendale, PA (1996)

84. C.H.P. Lupis and J.F. Elliott. Prediction of Enthalpy and Entropy Interaction Coefficients by the Central Atoms Theory. Acta Metallurgica, 15(2):265-276, 1967.

85. E.-H. Foo and C.H.P. Lupis. The Central Atoms Model of Multicomponent Interstitial Solutions and Its Applications to Carbon and Nitrogen in Iron Alloys. Acta Metallurgica, 21(10):1409-1430, 1973.

86. J. Lehmann, F. Bonnet, and M. Bobadilla. Thermodynamic Description of Liquid Steels and Metallurgical Slags by a Generalization of the Central Atoms Model. Iron \& steel technology, 3(6):115-123, 2006.

87. C. Chen, L. Zhang, and J. Lehmann. Thermodynamic Modelling of Phosphorus in Steelmaking Slags. High Temperature Materials and Processes, 32(3):237-246, 2013.

88. E.A. Lass, A. Zhu, G.J. Shiflet, and S.J. Poon. A Short-Range Ordering Description of Amorphous Metal Alloys Using the Central Atoms Model. Acta Materialia, 58(16):5460-5470, 2010. 